\title{
Apoptosis repressor with caspase recruitment domain enhances survival and promotes osteogenic differentiation of human osteoblast cells under Zoledronate treatment
}

\author{
LONGWEI HU $^{1 *}$, JING HAN $^{1 *}$, XI YANG $^{1}$, YANG WANG $^{1}$, HONGYA PAN $^{2}$ and LIQUN XU ${ }^{1}$ \\ ${ }^{1}$ Department of Oromaxillofacial Head and Neck Oncology, Shanghai Ninth People's Hospital, College of Stomatology; \\ ${ }^{2}$ Oral Bioengineering Laboratory/Regenerative Medicine Laboratory, Shanghai Research Institute of Stomatology, \\ Shanghai Key Laboratory of Stomatology, Shanghai Jiao Tong University School of Medicine, Shanghai 200011, P.R. China
}

Received September 25, 2015; Accepted June 23, 2016

DOI: $10.3892 / \mathrm{mmr} .2016 .5669$

\begin{abstract}
Zoledronate is one of the most potent nitrogen-containing bisphosphonates which has been demonstrated to result in osteoblast apoptosis and impact osteogenic differentiation in vitro. This effect of Zoledronate on osteoblasts may partially explain bisphosphonate-associated osteonecrosis of the jaw, a serious complication associated with treatment with bisphosphonates. Apoptosis repressor with caspase recruitment domain (ARC) is a multifunctional inhibitor of apoptosis that is physiologically expressed predominantly in post-mitotic cells such as cardiomyocytes, neurons and skeletal muscle cells. However, its effect on human osteoblasts remains unclear. The current study aimed to investigate the effects of ARC on human osteoblasts under the treatment of high concentrations of Zoledronate. ARC-overexpressed human osteoblasts were established and were exposed to Zoledronate with different concentrations $(0,1$ and $5 \mu \mathrm{M})$ in vitro. Cell numbers were detected using the MTT assay, and flow cytometry was used to identity cell apoptosis. Alkaline phosphatase staining, quantitative analysis and ectopic osteogenesis in nude mice were used to evaluate the osteogenic differentiation of ARC-overexpressed osteoblasts. It was observed that
\end{abstract}

Correspondence to: Dr Liqun Xu, Department of Oromaxillofacial Head and Neck Oncology, Shanghai Ninth People's Hospital, College of Stomatology, Shanghai Jiao Tong University School of Medicine, 639 Zhi-Zao-Ju Road, Shanghai 200011, P.R. China

E-mail:maxilla@sina.com

Mrs. Hongya Pan, Oral Bioengineering Laboratory/Regenerative Medicine Laboratory, Shanghai Research Institute of Stomatology, Shanghai Key Laboratory of Stomatology, Shanghai Jiao Tong University School of Medicine, 639 Zhi-Zao-Ju Road, Shanghai 200011, P.R. China

E-mail: paulin918@hotmail.com

*Contributed equally

Key words: Zoledronate, ARC, osteoblasts, osteogenic differentiation, apoptosis
ARC is able to reverse the inhibitory effect of Zoldronate on osteoblasts. ARC is additionally able to promote osteogenic differentiation of osteoblasts and inhibit their apoptosis. These observations suggest a critical role for ARC in the regulation of human osteoblasts under Zoledronate treatment.

\section{Introduction}

Bisphosphonates are currently the most widely used drugs for anti-resorptive therapy, and have been used for the treatment of metabolic bone diseases with increased bone resorption (1). Bisphosphonates represent a family of pyrophosphate analogues in which the oxygen linking the phosphates has been replaced by carbon. There are two major classes of bisphosphonates, the non-nitrogen-containing and nitrogen-containing, which have different molecular mechanisms of action (2). Among the currently used bisphosphonates, Zoledronate has been identified as one of the most potent nitrogen-containing bisphosphonates. Zoledronate can cause caspase-associated apoptosis in numerous cell types (3). It has been demonstrated that continuous exposure of osteoblasts to high-doses of Zoledronate can result in caspase-associated apoptosis of osteoblasts and thus inhibits osteoblasts function and/or survival (4). Short-term treatment with micromolar concentrations of Zoledronate may lead to a reductions of proliferation, mineralization, viability and alkaline phosphatase (ALP) activity in osteoblastic cell lines (5). Notably, based the results of a previous study, Zoledronate was identified to inhibit human osteoblast proliferation and osteogenic differentiation and lead to the apoptosis of osteoblasts in vitro (6).

Apoptosis repressor with caspase domain (ARC), also termed nol3, is an endogenous inhibitor of cell death that is highly expressed in cardiomyocytes, skeletal muscle cells and neurons in physiological conditions (7-9). It is able to inhibit extrinsic and intrinsic apoptosis pathways, and the mechanism of action includes inhibition of Fas-FADD binding and assembly of the death-inducing signaling complex, suppression of the activation of caspase- 2 and caspase- 8 and blocking of Bax activation (10-12). Elevated expression of ARC has been demonstrated to inhibit various modalities of apoptotic cell death signaling, while conversely loss of ARC sensitizes 
cells to apoptosis. However, it remains unclear whether this inhibitory effect of Zoledronate on osteoblasts can be reversed by ARC.

In the present study, the effect of ARC on anti-apoptosis of human osteoblasts was investigated under the treatment of high concentrations of Zoledronate. In addition, the effect of regulation of ARC was investigated on cell growth, osteogenic differentiation and new bone formation ability in osteoblast cells derived from the human jaw. It was identified that ARC was able to reverse the inhibitory effect of Zoledronate on osteoblasts, leading to an increase in the cell survival ability and osteogenic differentiation of human osteoblasts, and a reduction in apoptosis. The results of the current study may provide a novel strategy for the treatment of the side effects of Zoledronate on osteoblasts.

\section{Materials and methods}

Cell isolation and culture. The primary human osteoblasts were isolated and cultured as previously described (13). The sequential enzyme digestion method was used to obtain osteoblastic cells from excised alveolar bones of patients undergoing tooth extraction. The excised alveolar bones were first treated with $0.25 \%$ trypsin for $10 \mathrm{~min}$, and digested by $0.2 \%$ collagenase type II for $30 \mathrm{~min}$ and then $0.2 \%$ collagenase type II for $60 \mathrm{~min}$. The $0.25 \%$ trypsin was diluted by phosphate-buffered saline (PBS) and the $0.2 \%$ collagenase type II was diluted by Hank's balanced salt solution. Step one and step two digests were not used and the last digested cells were resuspended in Dulbecco's modified Eagle's medium (DMEM; Gibco; Thermo Fisher Scientific, Inc. Waltham, MA, USA). Fetal bovine serum (10\%; GE Healthcare Life Sciences, Logan, UT, USA), $100 \mathrm{U} / \mathrm{ml}$ streptomycin, $100 \mathrm{U} / \mathrm{ml}$ penicillin and 2 M L-glutamine (Sigma-Aldrich, St. Louis, MO, USA) were added into the DMEM. Cells were cultured in a humidified atmosphere of $37^{\circ} \mathrm{C}$ and $5 \% \mathrm{CO}_{2}$ for 2-4 days. Upon reaching confluence, cells were passaged for expansion and the second or third passages were used for the subsequent experiments. Each experiment was repeated 3 times using 3 different osteoblast strains isolated from 3 patients.

Lentiviral packaging and osteoblast transduction. For lentivrial packaging, human embryonic kidney $293 \mathrm{~T}$ (HEK293T) cells were cultured in 10-cm dishes until 90-95\% confluence. Recombinant virus plasmid pLV-nol3-enhanced green fluorescent protein (EGFP) and control vectors pLV-EGFP, together with packaging plasmids (pLP1, pLP2 and pLP/VSVG), were co-transfected into HEK293T cells using Lipofectamine 2000 (all from Genechem Co., Ltd., Shanghai, China).

Subsequent to $48 \mathrm{~h}$ of transduction, the supernatant of HEK293T cells which containing lentiviral particles was harvested and concentrated by passing through a $0.45-\mu \mathrm{m}$ filter. The concentrated lentiviral solutions of pLV-nol3-EGFP and pLV-EGFP were then added into cultured human osteoblasts at a variety of multiplicity of infections ranging from 0-100. After $72 \mathrm{~h}$, expression of EGFP was detected via fluorescence microscopy. Reverse transcription-quantitative polymerase chain reaction (RT-qPCR) and western blotting were used to analysis nol3 gene expression. 3-(4,5-dimethylthiazol-2-yl)-2,5-diphenyltetrazolium bromide (MTT) assay. The MTT assay was used to detect the number of viable cells which may further infer cell survival ability. Briefly, cells were seeded on a 96-well plate at a density of $5 \times 10^{3}$ cells/well and were cultured for $1,3,5$ and 7 days with fresh medium supplemented with various concentrations $(0$, 1 and $5 \mu \mathrm{M}$ ) of Zoledronate. At each time point, cells were washed twice with PBS and then incubated in $100 \mu 1$ DMEM supplemented with MTT $(5 \mathrm{mg} / \mathrm{ml})$ for $4 \mathrm{~h}$. Dimethyl sulfoxide were added into each well to dissolve the formazan crystals subsequent to removing the previous medium. The absorbance was read at the wavelength of $490 \mathrm{~nm}$ by a microplate reader (BioTek Instruments, Inc., Winooski, VT, USA) according to the manufacturer's instructions.

ALP staining and ALP activity assay. Cells were seeded at a density of 25,000 cells/well on 24-well plates and were treated with different concentrations $(0,1$ and $5 \mu \mathrm{M})$ of Zoledronate for 7 and 14 days. For ALP staining, cells were washed twice with PBS and then fixed with paraformaldehyde. After 15 min at $4^{\circ} \mathrm{C}$, paraformaldehyde was removed and cells were washed twice again with PBS and 5-bromo-4-chloro-3'-indolyphosphate p-toluidine salt/nitro-blue tetrazolium chloride solution (Beyotime Institute of Biotechnology, Shanghai, China) were added into each well, then incubated in the dark. Bicinchoninic acid assay (BCA) protein assay kit (Beyotime Institute of Biotechnology) were used to detect the ALP activities. ALP activities were normalized to total protein content and were expressed as percentage of total protein.

Cell apoptosis detection. Cells were treated with different concentrations of Zoledronate for 3 days, then the supernatant and adherent cells were collected for cell apoptosis analysis using centrifugation at $1,500 \mathrm{x}$ for $10 \mathrm{~min}$ at $4^{\circ} \mathrm{C}$. The BD Pharmingen $^{\text {TM }}$ APC Annexin V Apoptosis Detection kit (BD Biosciences, Franklin Lakes, NJ, USA) was used according to the manufacturer's instructions. Cells were washed twice with PBS and then suspended in $500 \mu$ l binding buffer, $5 \mu \mathrm{l}$ Annexin V-APC and $1 \mu \mathrm{l}$ propidium iodide (PI) solution were added into the binding buffer and incubated for $15 \mathrm{~min}$ in the dark at $37^{\circ} \mathrm{C}$. Cell apoptosis was analyzed by flow cytometry.

Gene expression analyzed by RT-qPCR. Cells were cultured under different concentrations of Zoledronate for 7 days, total RNA was extracted by TRIzol reagent (Invitrogen; Thermo Fisher Scientific, Inc.) and then reverse transcribed to cDNA with PrimeScript RT reagent kit (Takara Bio, Inc., Otsu, Japan). Specific gene primers were synthesized commercially and their sequences are listed in Table I. The Bio-Rad iQ5 real-time PCR system (Bio-Rad Laboratories, Inc., Hercules, CA, USA) and $10 \mu \mathrm{l}$ SYBR Premix Ex Taq kit (Takara Bio, Inc.) were used to amplify $1 \mu \mathrm{l}$ cDNA (in a reaction mixture with $0.5 \mu 1$ each primer and $8 \mu 1$ distilled water) and detect gene expression of ALP, bone sialoprotein (BSP), osteocalcin (OCN) and runt-related transcription factor 2 (Runx2). The thermocycling conditions were as follows: $95^{\circ} \mathrm{C}$ for $5 \mathrm{~min}$; and 40 cycles of $95^{\circ} \mathrm{C}$ for $10 \mathrm{sec}, 60^{\circ} \mathrm{C}$ for $30 \mathrm{sec}$. All relative gene expression values were normalized to $\beta$-actin based on the $2^{-\Delta \Delta} \mathrm{Cq}$ method (14). 
Table I. Sequences of the primers used for reverse transcription-quantitative polymerase chain reaction.

\begin{tabular}{lll}
\hline Genes & \multicolumn{1}{c}{ Forward primer } & \multicolumn{1}{c}{ Reverse primer } \\
\hline Actin & TGAAGTGTGACGTGGACATC & GGAGGAGCAATGATCTTGAT \\
ARC & ACAGCCTTGGGAAGTGAGAC & GTCCAGAGAGAAGCCAGACAA \\
OCN & GCGAGACATCAAGGAGAAGC & CCAATAAAGGAAGGCTGGAA \\
ALP & TCAAGCCAAACACAAACAGC & GGAGCCACAATCCAGTCATT \\
BSP & AAGGACGCTGGGAAATCTGT & GGGCATCTCGTTGTCTGAGT \\
Runx2 & GAAGAGGAGGAGGAAGAAGAGG & TCCATAGCCCAGTGTTGTAGC
\end{tabular}

ARC, apoptosis repressor with caspase recruitment domain; OCN, osteocalcin; ALP, alkaline phosphatase; BSP, bone sialoprotein; Runx2, runt-related transcription factor 2 .

Western blot analysis. For the western blot assay, cells were collected subsequent to being treated with different concentrations of Zoledronate for 7 days, and then they were lysed with a protein extraction reagent containing protease inhibitor cocktail, phosphatase inhibitor cocktail and phenyl-methanesulfonylfluoride (Shanghai Qianchen Technology Co., Ltd., Shanghai, China). The obtained protein concentrations were measured using the BCA protein assay kit (Beyotime Institute of Biotechnology) according to the manufacturer's instructions. Equal amounts $(36 \mu \mathrm{g})$ of protein from different samples were separated on sodium dodecyl sulfate-polyacrylamide gel electrophoresis and then transferred to polyvinylidene difluoride membranes. Membranes were incubated overnight at $4^{\circ} \mathrm{C}$ with the following primary antibodies: Rabbit anti-human ARC (1:1,000; Bioworld, Technology, Inc., St. Louis Park, MN, USA; cat. no. BS1018), rabbit anti-human cleaved caspase-3 (1:1,000; Cell Signaling Technology, Inc., Danvers, MA, USA; cat. no. 9664) and rabbit anti-human OCN (1:1,000; Cell Signaling Technology, Inc.; cat. no. 2890). Finally the membranes were visualized with horseradish peroxidase-conjugated goat anti-rabbit (Beyotime Institute of Biotechnology, Inc.) using the ECL Plus reagents (Amersham Pharmacia Biotech, USA) by UVItec Alliance 4.7 gel imaging system.

In-vivo osteogenic differentiation analysis. Cells were treated with 0 and $1 \mu \mathrm{M}$ Zoledronate for 3 days, seeded into calcium phosphate cement (CPC) constructs and then implanted subcutaneously into nude mice. A total of 8 male nude mice (BALB/c-nu; weight, 30-50 g; age, 4 weeks) were obtained from Shanghai SLAC Animal Laboratory Co., Ltd. (Shanghai, China), maintained under specific pathogen-free conditions and used in the experiment. The mice were housed separately with access to sterilized food and autoclaved water ad libitum. The environment was maintained at $24^{\circ} \mathrm{C}, 35-40 \%$ humidity with a $14 / 10-\mathrm{h}$ light/dark cycle. For each mouse, four dorsal subcutaneous pockets were made and one CPC construct was inserted into each pocket. A total of 32 constructs were divided into four groups: Group A, CPC/OB-nol3 $0 \mu \mathrm{M}$ Zoledronate $(\mathrm{n}=8)$; group $\mathrm{B}, \mathrm{CPC} / \mathrm{OB}$-con $0 \mu \mathrm{M}$ Zoledronate $(\mathrm{n}=8)$; group $\mathrm{C}$, CPC/OB-nol3 $1 \mu \mathrm{M}$ Zoledronate $(\mathrm{n}=8)$; and group $\mathrm{D}$, $\mathrm{CPC} / \mathrm{OB}$-con $1 \mu \mathrm{M}$ Zoledronate $(\mathrm{n}=8)$. After 10 weeks, the mice were sacrificed by cervical dislocation and the implants were removed, decalcified, paraffin-embedded, sectioned and hematoxylin and eosin-stained.

Photomicrographs were captured using a light microscope (Olympus Corporation, Tokyo, Japan) and Image Pro 5.0 software (Media Cybernetics, Inc., Rockville, MD, USA) was used to perform histomorphological analysis. New bone formation was defined as the percentage of observed new bone area in the entire implant (15). All of the animal and experimental protocols were approved by the Animal Research Committee of the Ninth People's Hospital Affiliated to Shanghai Jiao Tong University School of Medicine.

Statistical analysis. Results were expressed as mean \pm standard deviation. Independent sample t-tests were performed using SPSS software, version 11.0 (SPSS, Inc., Chicago, IL, USA) to determine statistically significant results. $\mathrm{P}<0.05$ was considered to indicate a statistically significant difference.

\section{Results}

ARC is stably expressed in human osteoblasts and can promote cell survival. The ARC-overexpressed osteoblasts were considered as the experimental groups and the empty vector transfected osteoblasts as the control groups. Three days subsequent to lentiviral infection, $>70 \%$ of osteoblasts were EGFP-positive as assessed by fluorescence microscopy (Fig. 1A). ARC expression in transfected osteoblasts was additionally detected by RT-qPCR and western blotting. As presented in Fig. 1B and C, ARC RNA and protein expression levels were significantly increased in ARC-overexpressed human osteoblasts when compared with the control group. In different concentrations of drug-treated ARC-overexpressed osteoblast groups, they both revealed significant increases in cell numbers in comparison with control groups from day 3-7 $(\mathrm{P}<0.05)$. The 0 and $1 \mu \mathrm{M}$-treated osteoblasts exhibited a continuous increase in growth and reached the plateau stage between days 5 and 7 in the ARC and control groups. In the $5 \mu \mathrm{M}$-treated groups, cell numbers were reduced from day 2 and the control group reduced more rapidly (Fig. 1C).

ARC inhibits apoptosis of human osteoblasts. High concentrations of Zoledronate lead to apoptosis of osteoblasts. In the current study, it was identified that ARC was able to reverse this effect and reduce cell apoptosis (Fig. 2). After 3 days of Zoledronate treatment, the percentages of apoptotic cells 
$\mathbf{A}$
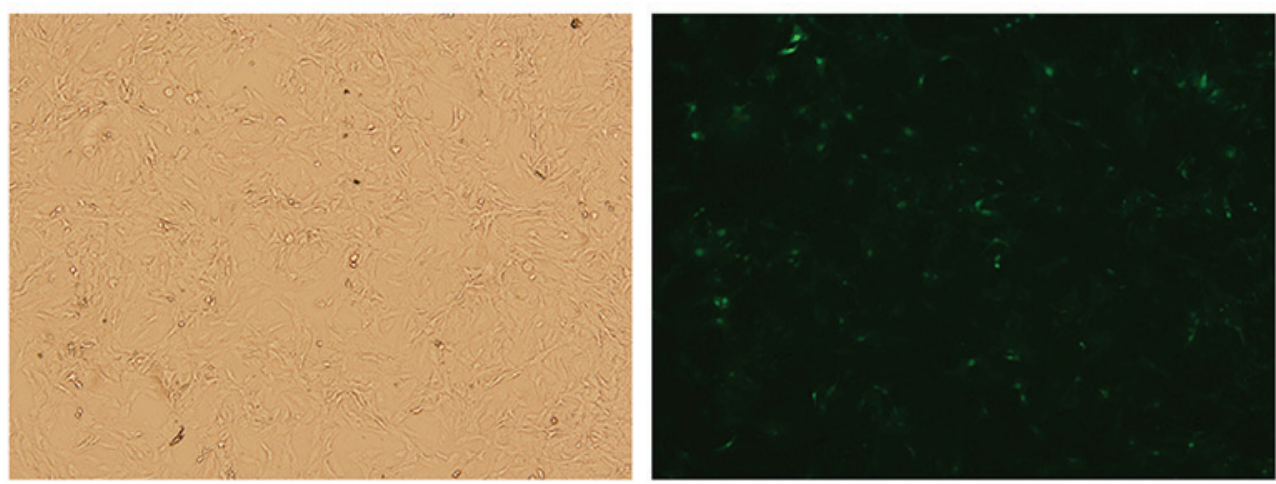

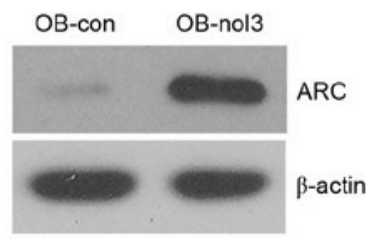

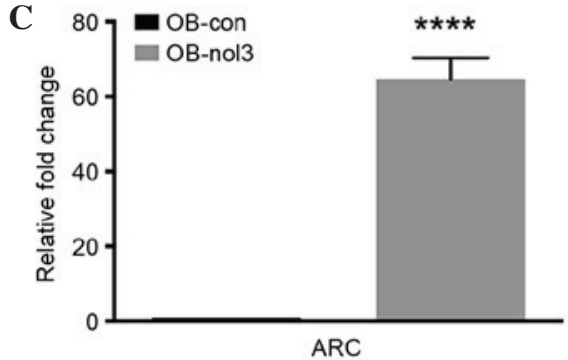

$\mathbf{D}$

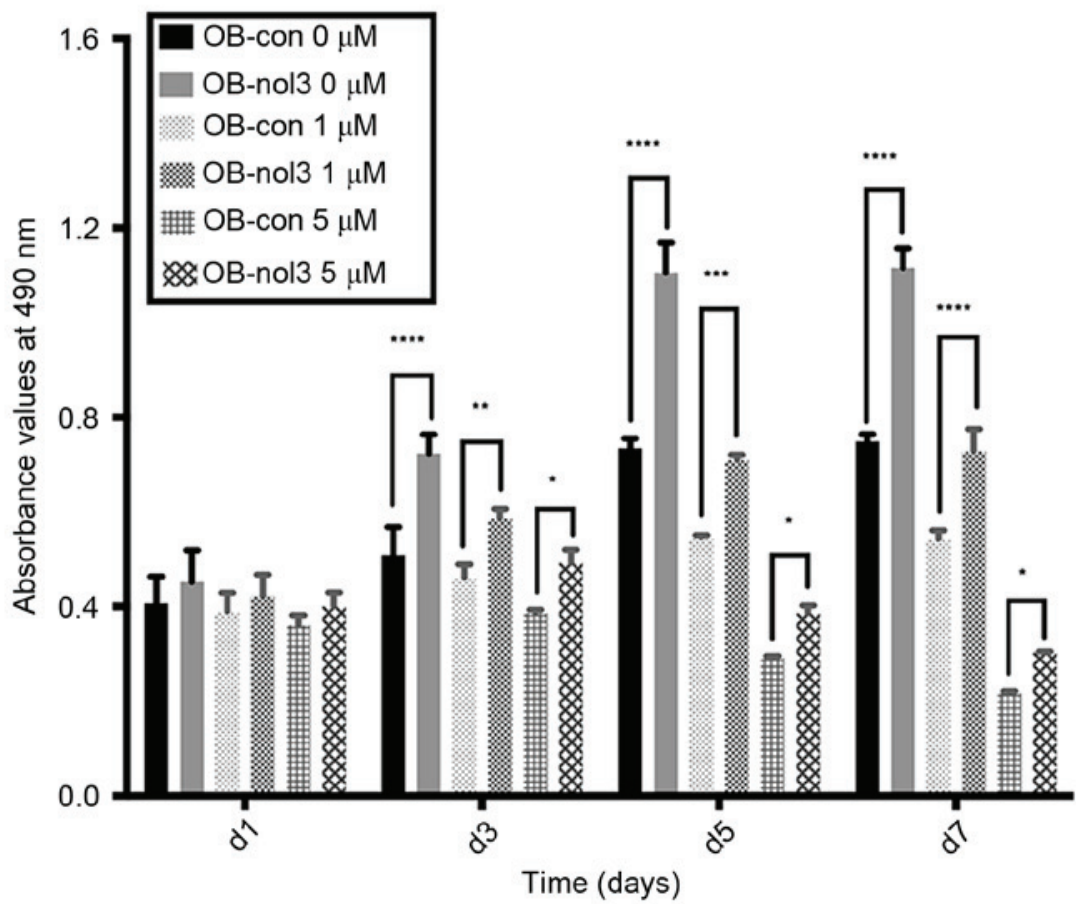

Figure 1. ARC overexpression in human osteoblasts by stable transduction and it can enhance survival of human osteoblasts. (A) The fluorescence microscopy detection of enhanced green fluorescent protein-positive human osteoblasts on day 3 subsequent to lentiviral infection (magnification, $\mathrm{x} 100$ ). (B) ARC mRNA and (C) protein expression levels were increased significantly in ARC-overexpressed cells compared with control cells. ${ }^{* * * *} \mathrm{P}<0.0001$ vs. control group. (D) Cell numbers [as measured by the 3-(4,5-dimethylthiazol-2-yl)-2,5-diphenyltetrazolium bromide assay] were significantly higher in ARC-overexpressed human osteoblasts compared with empty vector-transfected controls under 0,1 and $5 \mu \mathrm{M}$ concentrations of Zoledronate. ${ }^{*} \mathrm{P}<0.05,{ }^{* * *} \mathrm{P}<0.01,{ }^{* * * *} \mathrm{P}<0.001,{ }^{* * * * * *} \mathrm{P}<0.0001$ vs. empty vector-transfected control groups. ARC, apoptosis repressor with caspase recruitment domain; OB, osteoblast; con, control; nol, Zoledronate.

(including the early and later stages) were significantly reduced in ARC-overexpressed groups compared with the control groups $(\mathrm{P}<0.05$; Fig. $2 \mathrm{~B})$. In the $0 \mu \mathrm{M}$ drug-treated groups, the apoptosis percentages were 3.87 and $2.83 \%$ in control cells and ARC-overexpressed cells, respectively. Cell apoptosis percentages were increased to 13.35 and $8.15 \%$ in the $1 \mu \mathrm{M}$ treatment groups and 26.96 and $17.4 \%$ in $5 \mu \mathrm{M}$ treatment groups, respectively (Fig. 2A). The results of the western blot analysis identified a reduced production of the apoptosis marker protein cleaved-caspase-3 in ARC-overexpressed groups (Fig. 2C).

ARC promotes osteogenic differentiation of human osteoblasts. At day 7 post-Zoledronate treatment, ARC-overexpressed cells demonstrated stronger ALP-positive staining compared with 
A
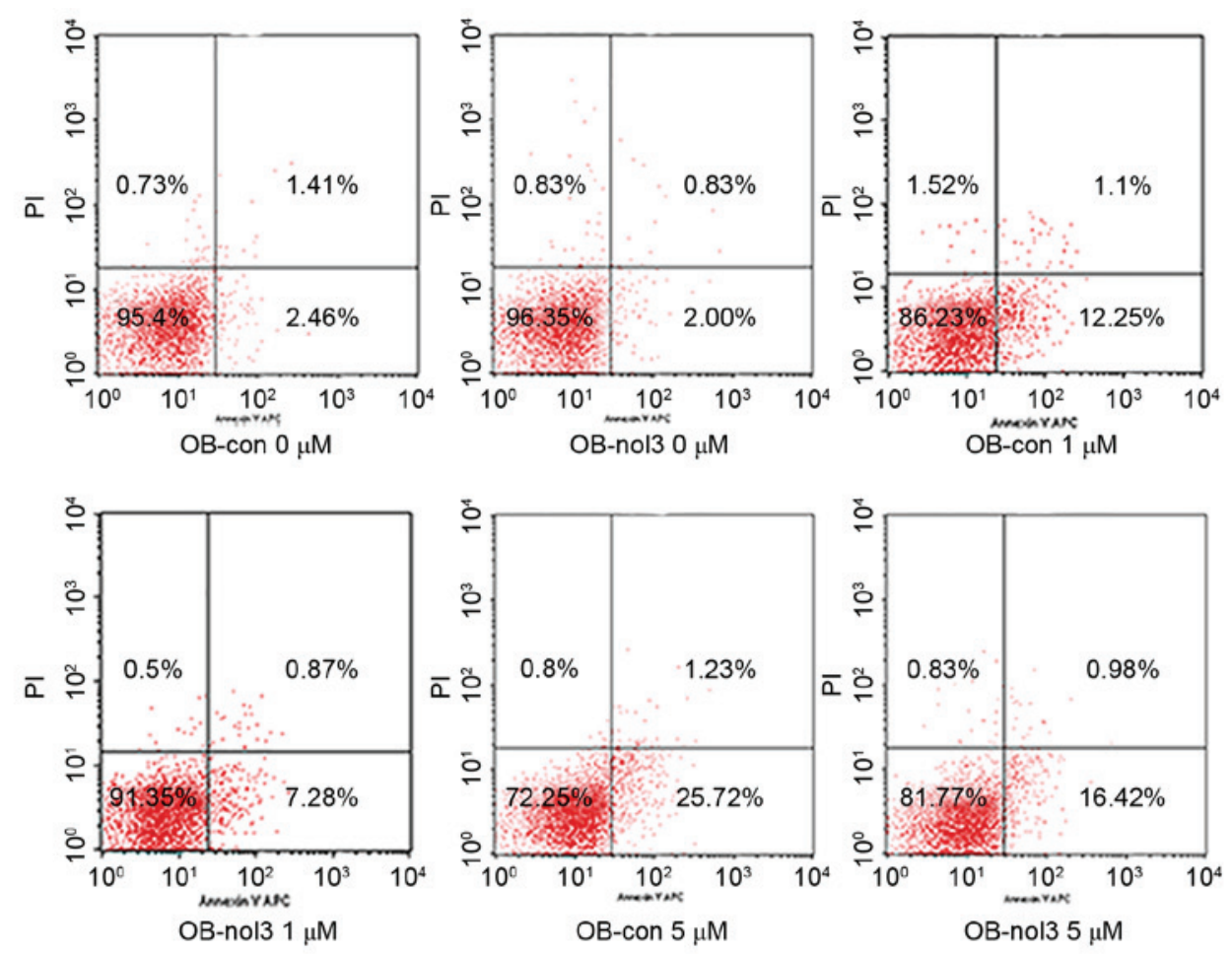

B

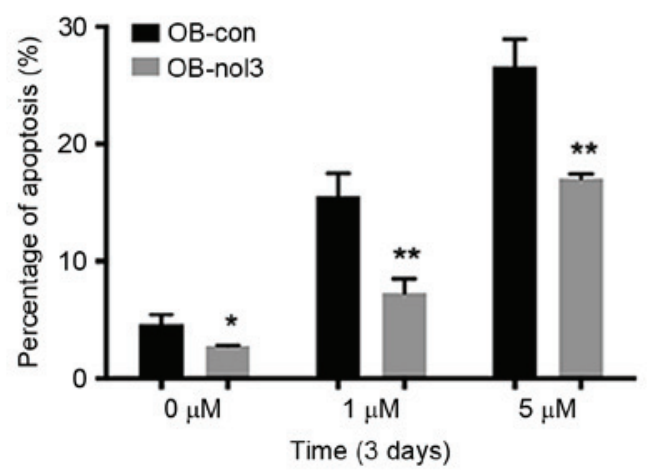

C

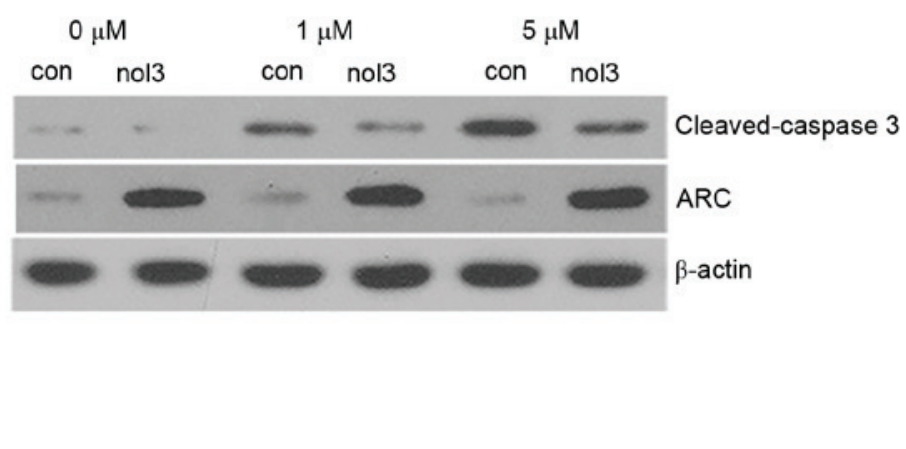

Figure 2. ARC inhibits apoptosis of human osteoblasts. (A) Cell apoptosis was detected by flow cytometry. One representative image from 3 independently performed experiments is presented. (B) Cell apoptosis was significantly reduced in ARC-overexpressed human osteoblasts compared with empty vector-transfected controls on day 3 under 0,1 and $5 \mu \mathrm{M}$ concentrations of Zoledronate treatment. ${ }^{*} \mathrm{P}<0.05,{ }^{* * *} \mathrm{P}<0.01$ vs. empty vector-transfected controls. (C) Western blot analysis identified reduced expression of cleaved-caspase-3 in ARC-overexpressed human osteoblasts compared with empty vector-transfected controls on day 7 under 0,1 and $5 \mu \mathrm{M}$ concentrations of Zoledronate treatment. ARC, apoptosis repressor with caspase recruitment domain; PI, propidium iodide; $\mathrm{OB}$, osteoblast; con, control; nol, Zoledronate.

control cells (Fig. 3A). In the $0 \mu \mathrm{M}$-treated group, ALP activity in the ARC-overexpressed cells increased to $112 \%$ of that of the control cells. In the 1 and $5 \mu \mathrm{M}$-treated groups, ALP activity was significantly increased to 136 and $118 \%$, respectively (Fig. 3B). Subsequent to 14 days of Zoledronate treatment, cell survival was $0 \%$ in the 1 and $5 \mu \mathrm{M}$-treated groups. However, the $0 \mu \mathrm{M}$-treated ARC-overexpressed cells exhibited stronger ALP-positive staining compared with control cells (Fig. 3C) and the ALP activity increased to $132 \%$ of that of the control cells (Fig. 3D). RT-qPCR results indicated a high expression level of ALP, BSP, OCN and Runx2 genes in ARC-overexpressed cells (Fig. 3E) and western blot analysis confirmed high expression levels of OCN in ARC-overexpressed osteoblasts (Fig. 3F).
In vivo osteogenic potential of ARC overexpressed osteoblasts and control osteoblasts. At 10 weeks subsequent to implantation, histological examinations were conducted for four groups under light microscopy. In osteoblasts treated with 0 and $1 \mu \mathrm{M}$ Zoledronate, increased new bone formation was observed in the $\mathrm{CPC} / \mathrm{OB}$-nol3 groups in comparison with $\mathrm{CPC} / \mathrm{OB}$-con groups, respectively (Fig. 4A). Histomorphometrical analysis further revealed that in the $0 \mu \mathrm{M}$ Zoledronate-treated groups, the percentage of new bone area in the CPC/OB-nol3 group increased to $149.6 \%$ of the control group, and in $1 \mu \mathrm{M}$-treated groups, the percentage of new bone area in the CPC/OB-nol3 group increased to $144.9 \%$ of the control group (Fig. 4B). These in vivo results further indicated that the ARC-overexpressed 
A

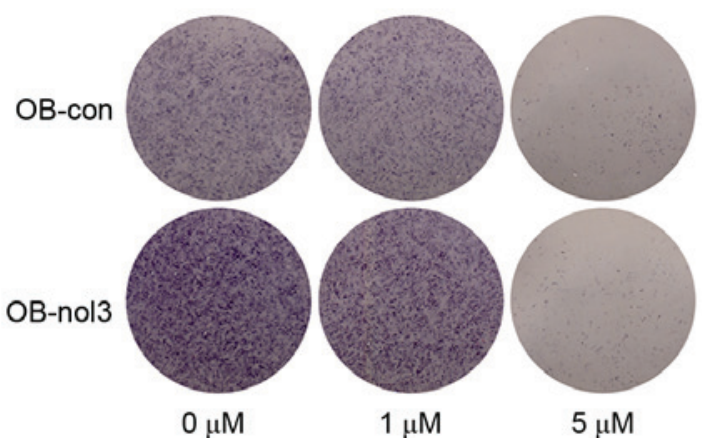

C

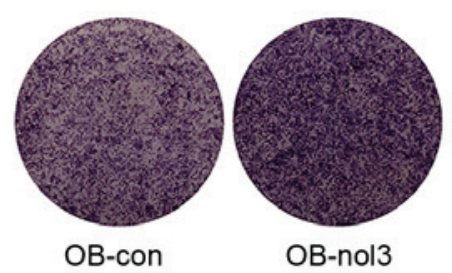

B

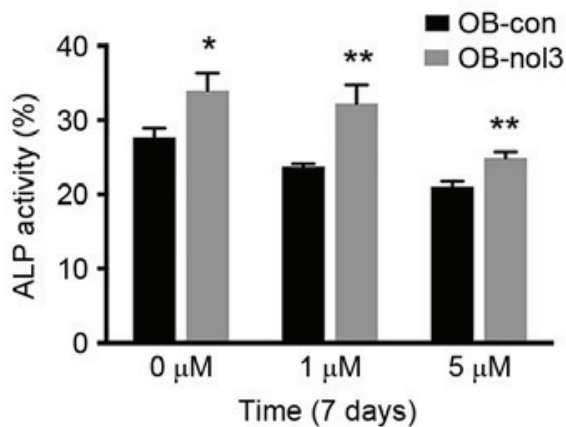

D

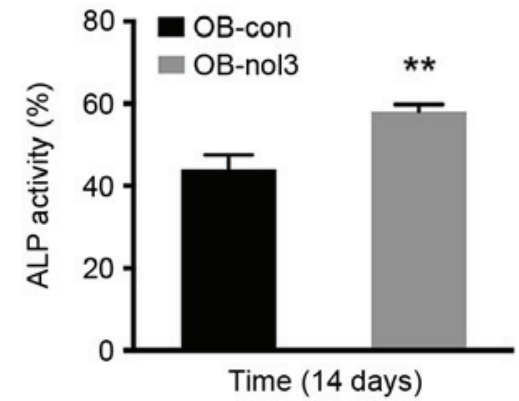

E
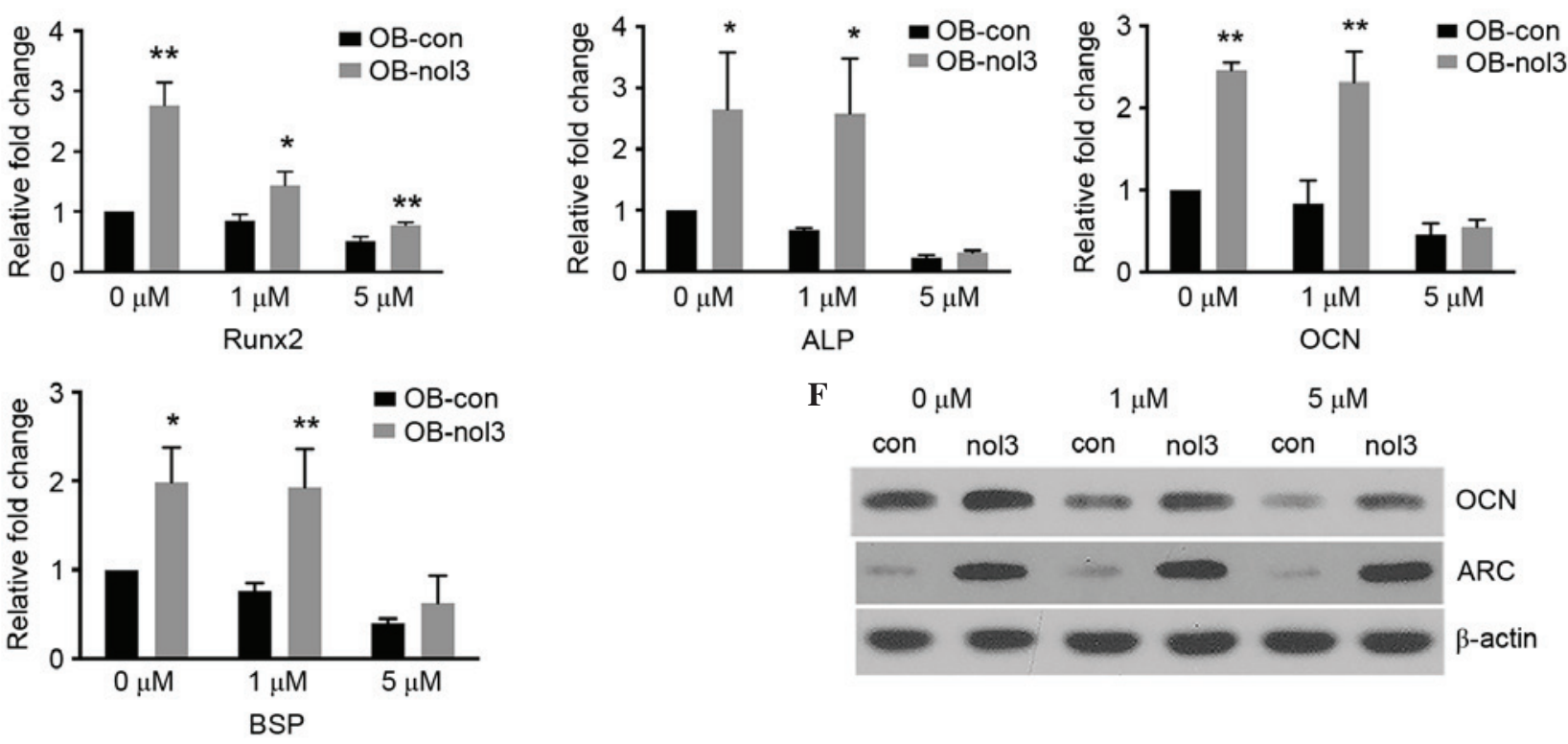

F

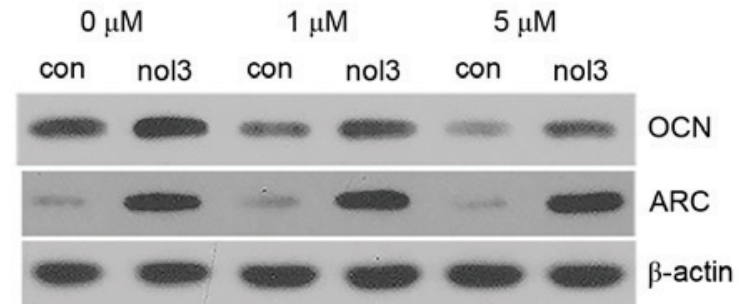

Figure 3. ARC promotes osteogenic differentiation of human osteoblasts. (A) ALP staining and (B) ALP activity of ARC-overexpressed human osteoblasts and empty vector-transfected controls at day 7. (C) ALP staining and (D) ALP activity of ARC-overexpressed human osteoblasts and empty vector-transfected controls at day 14. The ALP activity was significantly upregulated in ARC-overexpressed human osteoblasts compared with empty vector-transfected controls under 0,1 and $5 \mu \mathrm{M}$ concentrations of Zoledronate treatment. (E) Reverse transcription-quantitative polymerase chain reaction analysis of the expression of ALP, BSP, OCN and Runx2 at day 7. The expression levels were significantly increased in ARC-overexpressed human osteoblasts compared with empty vector-transfected controls under 0 and $1 \mu \mathrm{M}$ concentrations of Zoledronate treatment. While in the $5 \mu \mathrm{M}$ drug-treated groups, no significant differences between ARC-overexpressed human osteoblasts and empty vector-transfected controls were observed in the ALP, BSP and OCN expression levels. "P $<0.05$, ${ }^{* *} \mathrm{P}<0.01$ vs. empty vector-transfected controls. (F) Western blot analysis identified that increased expression of OCN in ARC-overexpressed human osteoblasts compared with empty vector-transfected controls on day 7 under 0,1 and $5 \mu \mathrm{M}$ concentrations of Zoledronate treatment. ARC, apoptosis repressor with caspase recruitment domain; ALP, alkaline phosphatase; BSP, bone sialoprotein; OCN, osteocalcin; Runx2, runt-related transcription factor 2; OB, osteoblast; con, control; nol, Zoledronate.

osteoblasts exhibited higher osteogenic potential compared with the control cells.

\section{Discussion}

Zoledronate is one of the most potent nitro-containing bisphosphonates which is widely used in the clinic for antiresorptive therapies, and has been demonstrated to cause osteoblast apoptosis in vitro. Zoledronate acid can inhibit the synthases of the enzymes farnesyl and geranylgeranyl diphosphate and further lead to the substrate and upstream accumulation of isoprenoid pyrophosphate lipids. The enzymes farnesyl and geranylgeranyl diphosphate are important parts of the mevalonate pathway and their inhibi- 
A
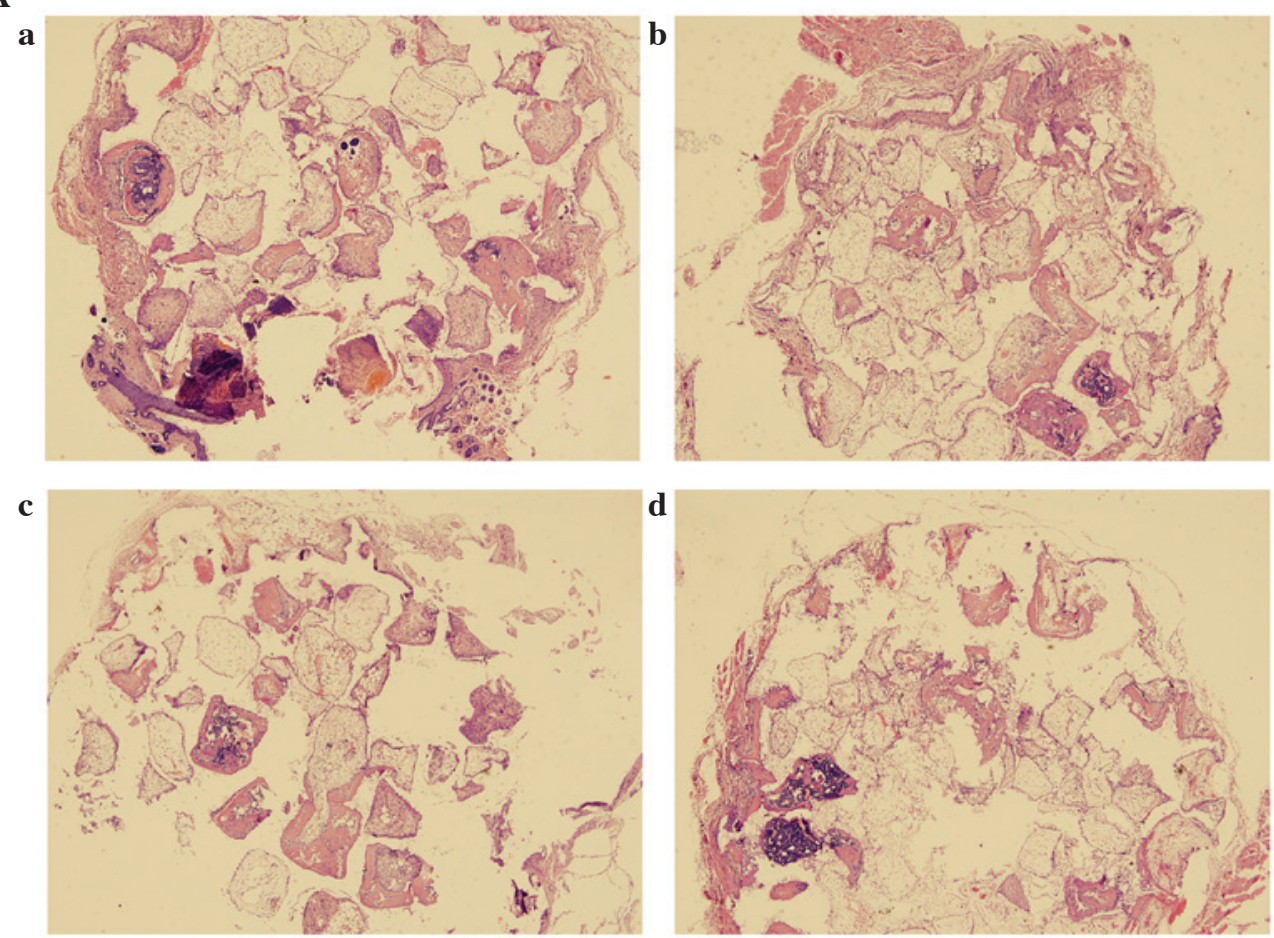

B

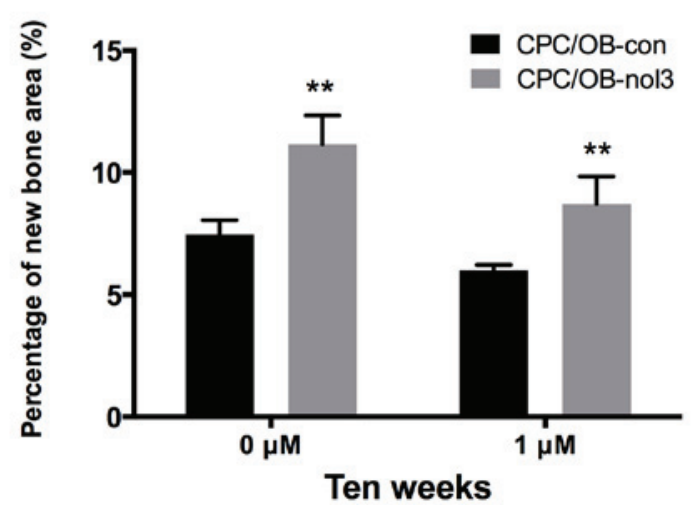

Figure 4. (A) Histological observations at 10 weeks subsequent to implantation (magnification, $\mathrm{x} 100$ ). (a) $0 \mu \mathrm{M} Z$ Zoledronate $\mathrm{CPC} / \mathrm{OB}$-nol3; (b) $0 \mu \mathrm{M}$ Zoledronate CPC/OB-con; (c) $1 \mu \mathrm{M}$ Zoledronate CPC/OB-nol3; (d) $1 \mu \mathrm{M}$ Zoledronate CPC/OB-con. Newly formed bone was observed in the CPC/OB-nol3 groups (a and c) and the CPC/OB-con groups (b and d). (B) The percentages of new bone area were significantly increased in the CPC/OB-nol3 groups compared with the $\mathrm{CPC} / \mathrm{OB}$-con groups by histomorphometric analysis ( ${ }^{* *} \mathrm{P}<0.01 \mathrm{vs}$. CPC/OB-con groups). CPC, calcium phosphate cement; OB, osteoblast; nol, Zoledronate; con, control.

tions can disrupt the prenylation of guanosine triphosphate binding proteins Ras and Rho, and further influence the subcellular distribution of these proteins $(16,17)$. This accompanied by downstream events can lead to caspase-associated apoptosis in osteoclasts and any other cell types in vitro, including osteoblasts, mesenchymal precursor cells and in addition tumor cells using $\mu \mathrm{M}$ concentrations $(18,19)$. This effect of Zoledronate on osteoblasts may partially explain bisphosphonate-associated osteonecrosis of the jaw (BRONJ), the first described long-term complication associated with Zoledronate treatment (20). Trauma is a risk factor for the occurrence of BRONJ. Bisphosphonates are able to tightly bind to hydroxyapatite and they can be continuously released from the traumatic lesions, which may lead to high concentrations of drugs in the traumatic sites. Thus $\mu \mathrm{M}$ concentrations of bisphosphonates that are comparable to in-vivo concentrations in the traumatic sites were used to treat osteoblasts in vitro (21). The tested concentrations of Zoledronate were additionally based on preliminary experiments which enabled us to exclude the levels that cause rapid cell death. As an anti-apoptotic suppressor, ARC is highly expressed in terminally differentiated cells and diverse types of cancer including pancreatic, colorectal, breast, lung, cervical and prostate cancer (22-24). In the present study, the effects of ARC on osteoblasts derived from human jaw under Zoledronate treatment were investigated.

In the present study, ARC-overexpressed osteoblasts were established, and they were exposed to 0,1 and $5 \mu \mathrm{M}$ concentrations of Zoledronate. Subsequent to 3 days of culture, it was identified that the ARC-overexpressed groups exhibited reduced cell apoptosis compared with control groups, which was verified by the increased expression levels of the cleaved-caspase-3 protein (Fig. 2C). This process is suggested to be predominantly due to the fact that ARC is able 
to block the caspase-associated apoptosis pathways caused by Zoledronate treatment.

ARC can also enhance osteoblast cell survival (Fig. 1C) and osteogenic differentiation (Fig. 3). In the current study, it was identified that the osteogenic markers ALP, Runx2, OCN and BSP, in addition to ALP activity, were significantly increased in ARC-overexpressing groups compared with the control groups. Ectopic new bone formation in nude mice was additionally observed, and the results revealed that bone formation was increased in ARC-overexpressed osteoblasts. The cells in the $5 \mu \mathrm{M}$ Zoledronate-treated groups were not set due to the fact that the cells exhibited lower rates of vitality following 3 days of $5 \mu \mathrm{M}$ drug treatment. Taken together, these results suggested that ARC is able to promote osteogenic differentiation, however, the exact mechanism is unclear and requires further exploration.

In conclusion, the present study revealed a previously unknown role of ARC in the regulation of cell apoptosis and osteogenic differentiation of osteoblasts under different concentrations of Zoledronate treatment. Briefly, ARC increases osteoblast anti-apoptotic effect and promotes osteoblast osteogenic differentiation. Thus, the observations of the present study offer novel insight into the role of ARC in osteoblast survival and differentiation. ARC may be considered as a promising agent to block the toxic effects of Zoledronate on osteoblasts, and thus may be applicable to rescue BRONJ. However, this effect requires further study.

\section{Acknowledgements}

The current study was funded by the National Natural Science Foundation of China (grant no. 81400482), and Shanghai Jiao Tong University Medical Engineering Cross Research Fund (grant no. YG2012MS42).

\section{References}

1. Lewiecki EM: Safety of long-term bisphosphonate therapy for the management of osteoporosis. Drugs 71: 791-814, 2011.

2. Baron R, Ferrari S and Russell RG: Denosumab and bisphosphonates: Different mechanisms of action and effects. Bone 48: 677-692, 2011.

3. Senaratne SG, Mansi JL and Colston KW: The bisphosphonate zoledronic acid impairs Ras membrane [correction of impairs membrane] localisation and induces cytochrome $\mathrm{c}$ release in breast cancer cells. Br J Cancer 86: 1479-1486, 2002.

4. Kaiser T, Teufel I, Geiger K, Vater Y, Aicher WK, Klein G and Fehm T: Bisphosphonates modulate vital functions of human osteoblasts and affect their interactions with breast cancer cells. Breast Cancer Res Treat 140: 35-48, 2013.

5. Tenta R, Sourla A, Lembessis P and Koutsilieris M: Bone-related growth factors and zoledronic acid regulate the PTHrP/PTH.1 receptor bioregulation systems in MG-63 human osteosarcoma cells. Anticancer Res 26: 283-291, 2006.

6. Hu L, Han J, Pan H and Xu L: High concentration of zoledronate inhibits osteogenesis differentiation and promotes apoptosis of human osteoblasts. China Journal of Stomatology 24: 389-394, 2015 .
7. Li Y, Ge X and Liu X: The cardioprotective effect of postconditioning is mediated by ARC through inhibiting mitochondrial apoptotic pathway. Apoptosis 14: 164-172, 2009.

8. Li YZ, Liu XH, Zhu XM and Cai LR: ARC contributes to the inhibitory effect of preconditioning on cardiomyocyte apoptosis. Apoptosis 12: 1589-1595, 2007.

9. Hong YM, Jo DG, Lee JY, Chang JW, Nam JH, Noh JY, Koh JY and Jung YK: Down-regulation of ARC contributes to vulnerability of hippocampal neurons to ischemia/hypoxia. FEBS Lett 543: 170-173, 2003.

10. Koseki T, Inohara N, Chen S and Núñez G: ARC, an inhibitor of apoptosis expressed in skeletal muscle and heart that interacts selectively with caspases. Proc Natl Acad Sci USA 95: 5156-5160, 1998.

11. Jo DG, Jun JI, Chang JW, Hong YM, Song S, Cho DH, Shim SM, Lee HJ, Cho C, Kim DH and Jung YK: Calcium binding of ARC mediates regulation of caspase 8 and cell death. Mol Cell Biol 24: 9763-9770, 2004.

12. Brenner D and Mak TW: Mitochondrial cell death effectors. Curr Opin Cell Biol 21: 871-877, 2009.

13. Aino M, Nishida E, Fujieda Y, Orimoto A, Mitani A, Noguchi T, Makino H, Murakami S, Umezawa A, Yoneda T and Saito M: Isolation and characterization of the human immature osteoblast culture system from the alveolar bones of aged donors for bone regeneration therapy. Expert Opin Biol Ther 14: 1731-1744, 2014.

14. Livak KJ and Schmittgen TD: Analysis of relative gene expression data using real-time quantitative PCR and the 2(-Delta Delta C(T)) method. Methods 25: 402-408, 2001.

15. Wang XJ, Huang H, Yang F, Xia LG, Zhang WJ, Jiang XQ and Zhang FQ: Ectopic study of tissue-engineered bone complex with enamel matrix proteins, bone marrow stromal cells in porous calcium phosphate cement scaffolds, in nude mice. Cell Prolif 44: 274-282, 2011.

16. Wiemer AJ, Tong H, Swanson KM and Hohl RJ: Digeranyl bisphosphonate inhibits geranylgeranyl pyrophosphate synthase. Biochem Biophys Res Commun 353: 921-925, 2007.

17. Xing LP and Boyce BF: Regulation of apoptosis in osteoclasts and osteoblastic cells. Biochem Biophys Res Commun 328: 709-720, 2005

18. Ebert R, Zeck S, Krug R, Meissner-Weigl J, Schneider D, Seefried L, Eulert J and Jakob F: Pulse treatment with zoledronic acid causes sustained commitment of bone marrow derived mesenchymal stem cells for osteogenic differentiation. Bone 44: 858-864, 2009.

19. Daubiné F, Le Gall C, Gasser J, Green J and Clézardin P: Antitumor effects of clinical dosing regimens of bisphosphonates in experimental breast cancer bone metastasis. J Natl Cancer Inst 99: 322-330, 2007.

20. Manzano-Moreno FJ, Ramos-Torrecillas J, De Luna-Bertos E, Ruiz C and García-Martínez O: High doses of bisphosphonates reduce osteoblast-like cell proliferation by arresting the cell cycle and inducing apoptosis. J Craniomaxillofac Surg 43: 396-401, 2015.

21. Cheong S, Sun S, Kang B, Bezouglaia O, Elashoff D, McKenna CE, Aghaloo TL and Tetradis S: Bisphosphonate uptake in areas of tooth extraction or periapical disease. J Oral Maxillofac Surg 72: 2461-2468, 2014.

22. Chen LH, Jiang CC, Watts R, Thorne RF, Kiejda KA, Zhang XD and Hersey P: Inhibition of endoplasmic reticulum stress-induced apoptosis of melanoma cells by the ARC protein. Cancer Res 68: 834-842, 2008

23. Mercier I, Vuolo M, Jasmin JF, Medina CM, Williams M, Mariadason JM, Qian H, Xue X, Pestell RG, Lisanti MP and Kitsis RN: ARC (apoptosis repressor with caspase recruitment domain) is a novel marker of human colon cancer. Cell Cycle 7: 1640-1647, 2008

24. Wang M, Qanungo S, Crow MT, Watanabe M and Nieminen AL: Apoptosis repressor with caspase recruitment domain (ARC) is expressed in cancer cells and localizes to nuclei. FEBS Lett 579: 2411-2415, 2005. 